\title{
Orbital cellulitis following Caldwell Luc procedure for maxillary sinusitis leading to a rare complication of central retinal artery occlusion
}

Deepti Mahajan, Gaurav Sharma, Rajeev Tuli

Department of Ophthalmology, Rajendra Prasad Medical College, Kangra, India

\begin{abstract}
Posterior orbital cellulitis is a clinical syndrome in which early severe visual loss overshadows or precedes accompanying inflammatory orbital signs. It is an ocular emergency that can threaten life and vision. Central retinal artery occlusion (CRAO) is a very rare condition leading to sudden onset of decreased vision and can even progress to blindness. We report a case of orbital cellulitis following Caldwell Luc surgery in a middle-aged diabetic woman, resulting in the rare complication of CRAO, leading to blindness in her right eye. The development of retinal artery occlusion after orbital cellulitis has not been well documented in literature. A quick diagnosis and close monitoring following sinus surgery is therefore needed to prevent such rare complications to happen.
\end{abstract}

Keywords: artery, Caldwell Luc, cellulitis, occlusion, orbital, retina, sinusitis

\section{Introduction}

Central retinal artery occlusion (CRAO) is a very rare condition with an incidence of $1 / 10,000$ of outpatient visits. ${ }^{1}$ This event is sudden and devastating, leading to decreased visual acuity. ${ }^{2}$ Visual loss following orbital cellulitis can still occur despite prompt diagnosis and management. Acute arterial occlusion is an unusual but known complication of orbital cellulitis. ${ }^{3}$ Thus, it is one of the most important conditions in ophthalmology that warrants immediate intervention. We report a rare case of CRAO with posterior orbital cellulitis in a patient operated by Caldwell Luc surgery for maxillary sinusitis.

\section{Case}

A 55-year-old diabetic female presented to the ENT outpatient department on 27 March, 2017 with the chief complaint of swelling in the right cheek since 4 days. She was diagnosed with maxillary sinusitis with periorbital cellulitis. On $31 \mathrm{March}$, 2017, an eye consultation was requested. Visual acuity was 6/12 in the right and

Correspondence: Dr Deepti Mahajan, Senior Resident, Department of

Ophthalmology, Rajendra Prasad Medical College, Kangra, Himachal Pradesh, India.

E-mail:mahajandeepti22@gmail.com 
left eye. Ocular movements were normal. Fundus examination revealed a normal disc and there were no clinical features suggestive of preseptal or orbital cellulitis. A computed tomography (CT) scan was done, which revealed opacification of the maxillary sinus (right side $>$ left side) with diffuse inflammatory changes in the soft tissue of the right cheek (Fig. 1). Injection ceftriaxone and injection metronidazole was started intravenously. After a complete work up, Caldwell Luc operation was done for pus removal from the maxillary sinus. The patient was given intravenous injections ceftriaxone, metronidazole and paracetamol and tablet levocetirizine. Two days following surgery, the patient reported to the eye department with diminished vision in the right eye. She denied perception of light in the right eye. Fundus examination of the right eye revealed disc pallor, cattle tracking of the vessels in the superior arcade. There was evidence of ischaemic retina (Fig. 2). Left eye fundus examination was normal. Intraocular pressure (IOP) in the right and left eye was $13 \mathrm{~mm} \mathrm{Hg}$. A diagnosis of CRAO was made. Ocular massage was done for 5 minutes and investigations were done. Fasting blood sugar was $154 \mathrm{mg}$, blood profile was normal, ESR was $12 \mathrm{~mm} / \mathrm{h}$, electrocardiogram revealed a normal study, carotid Doppler revealed mild soft-tissue plaque in the right carotid artery, $0.2 \mathrm{~cm}$ in thickness with the rest of the vessels being normal. ECHO showed left-ventricular diastolic dysfunction grade 1. Fungal culture and stain of

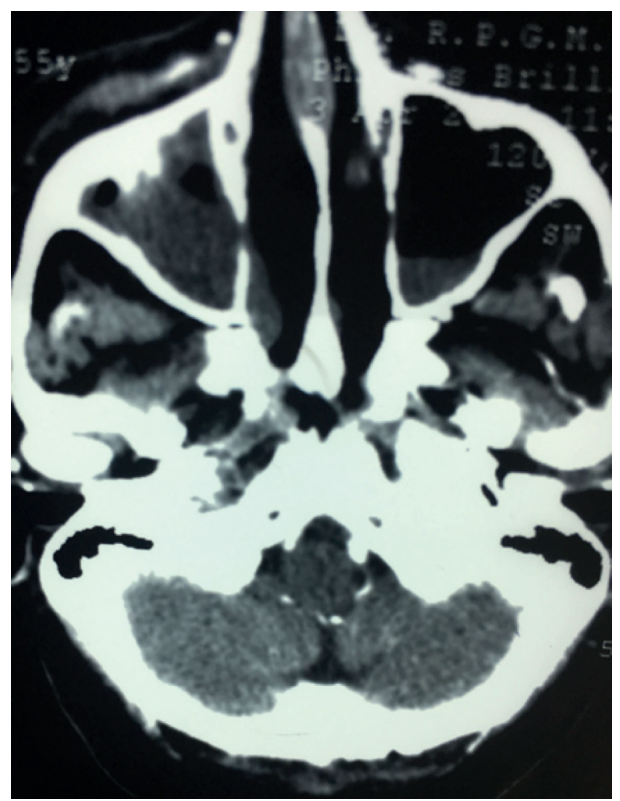

Fig. 1. CT scan of nose and paranasal sinuses showing opacification of the maxillary sinus, right side $>$ left side. 


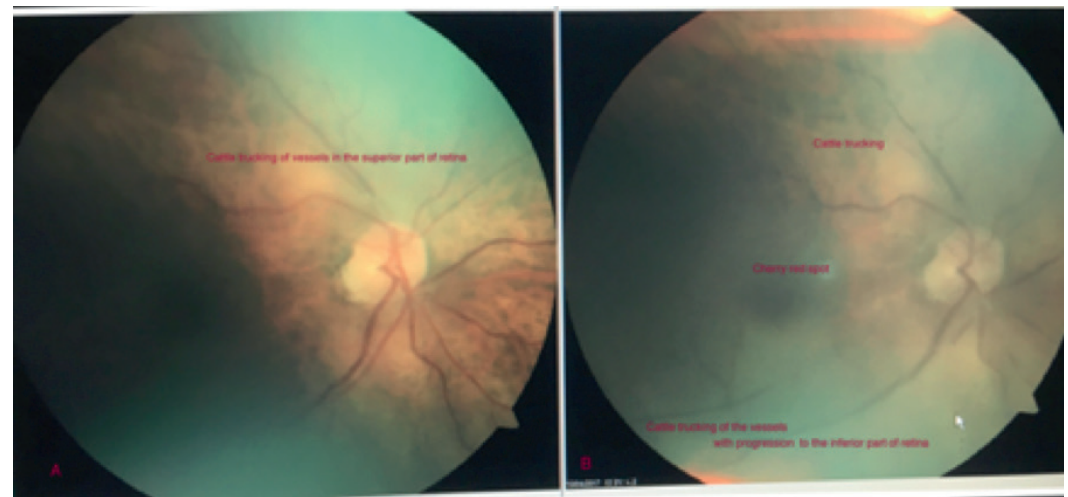

Fig. 2. Fundus photograph of right eye showing progression of ischemic changes in the retina. (Left) Cattle trucking of the vessels. (Right) Ischemic changes in the fundus.

pus from the sinus was negative. CT head revealed symmetrical prominence of the ventricular system.

On 12 April, 2017, she denied perception of light, and fundus of the right eye (Fig. 2) revealed increased cattle tracking along the vessels and a cherry-red spot at the macula. The chemosis in the right eye had increased, pupil in the right eye was non-reacting to light and ocular movements had restricted (Fig. 3).

\section{Discussion}

CRAO is mostly caused by a thrombus or embolus that leads to reduced blood perfusion of the retina. ${ }^{4} \mathrm{It}$ is a very rare complication of sinus surgery complicated with orbital cellulitis. It clinically presents as sudden painless acute unilateral or bilateral vision loss in the range of counting fingers to no light perception. The rate of spontaneously recanalization of the artery is about $15 \%$ with timely intervention. ${ }^{5}$ The prognosis is very poor as only $61 \%$ of patients can regain a final VA of $6 / 120$ or less. ${ }^{3}$ One of the typical findings in CRAO is cherry-red spot that is found in about $90 \%$ of cases.

Sinus surgery leading to ophthalmic complications is not very common, despite the proximity of the orbit to the paranasal sinuses. ${ }^{6}$ The most frequent complication of the Caldwell Luc operation is reported to be the paraesthesia or anaesthesia of the inferior orbital nerve. Blindness is a rare complication, which occurs due to direct trauma to the optic nerve or the pressure effect of an orbital haemorrhage. ${ }^{7}$ Buus and co-workers detected orbital complications in only seven of their patients in a period of 10 years of their study. ${ }^{6}$ Griffiths and Smith observed blindness and ocular motility disturbance in two patients following the Caldwell Luc procedure. ${ }^{8}$ If retinal ischemia persists for more than 100 minutes, recovery of vision is unusual. ${ }^{7}$ 


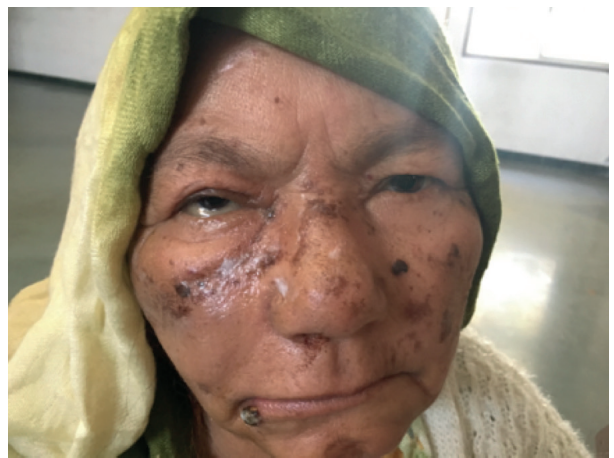

Fig. 3. Increased chemosis of the right eye with restriction of extraocular movements.

Many theories are given to explain the reason why CRAO occurs during surgical procedures. A prolonged hypotensive status with reduction in blood flow or increasing intraocular pressure including ocular compression during certain surgical procedures is associated with ocular ischemia, ${ }^{8}$ which also causes ischemia of the retina, leading to CRAO and vision loss after surgery.

Ocular vascular damage during surgical maneuvers results in activation and aggregation of platelets and activated platelets release serotonin, which is a vasoconstrictor and induces a transient arterial spasm, causing transient or complete arterial occlusion, resulting in ischemia of retina leading to CRAO. ${ }^{9}$

Acute visual loss may be associated with acute sinusitis either secondary to complicated orbital cellulitis or as a part of the orbital apex syndrome. El-Sayed and Al-Muhaimeid ${ }^{10}$ reported two cases of acute visual loss as a complication of orbital cellulitis due to sinusitis. One patient showed improvement in vision from hand motion to normal vision after intravenous treatment of pansinusitis and associated orbital cellulitis. The second patient recovered vision from no light perception to normal levels after exploration of the sphenoid and ethmoid sinuses along with intravenous antibiotics.

Slavin and Glaser ${ }^{11}$ described three cases of sphenoethmoiditis causing permanent visual loss associated with minimal signs of orbital inflammation. This entity was called 'posterior orbital cellulitis'. Slavin and Glaser ${ }^{11}$ defined it as a clinical syndrome in which early severe visual loss overshadows or precedes accompanying inflammatory orbital signs. Acute blindness may also result from orbital infarction syndrome. Orbital infarction is a disorder that may occur secondary to different mechanisms: (a) acute perfusion failure, common carotid artery occlusion; (b) systemic vasculitis and (c) orbital cellulitis with vasculitis, mucurmycosis. ${ }^{11}$

There is a critical time of 90 to 120 minutes after occlusion to perform interventions to improve vision; however, there is no approved modality to be effective 
in the treatment of CRAO. ${ }^{12}$ Immediate ocular massage and anterior chamber paracentesis, use of drugs such as intravenous acetazolamide and mannitol, inhalation of a mixture of $95 \%$ oxygen and $5 \%$ carbon dioxide (carbogen) are given to reduce IOP and improve blood flow to the eye.

\section{Conclusion}

Anatomic knowledge, proper evaluation, early recognition of complications and appropriate management of patients undergoing Caldwell Luc surgery will minimize these rare complications. Normally, orbital cellulitis responds to systemic antibiotic therapy and surgical drainage without signs of optic nerve compromise. We recommend that in all patients undergoing surgery by Caldwell Luc procedure, the ocular examination should be performed immediately after surgery, with a close follow up after the surgery to rule out the rare complication of CRAO, as delay in diagnosis and intervention could eventuate to severe vision loss.

\section{Acknowledgements}

The authors would like to thank the entire Department of Ophthalmology at Rajendra Prasad Medical College in Kangra, India which worked as a team for doing various procedures for the patients.

\section{References}

1. Hayreh SS. Acute retinal arterial occlusive disorders. Prog Retin Eye Res. 2011;30:359-394.

2. Brown GC, Magargal LE. Central retinal artery obstruction and visual acuity. Ophthalmology. 1982;89:14-19.

3. Jarrett WH, Gutman FA. Ocular complications of infection in the paranasal sinuses. Arch Ophthalmol. 1969;81:683-688.

4. Chen CS, Lee AW. Management of acute central retinal artery occlusion. Nat Clin Pract Neurol. 2008;4:376-383.

5. Hayreh SS, Podhajsky P. Ocular neovascularization with retinal vascular occlusion. II. Occurrence in central and branch retinal artery occlusion. Arch Ophthalmol. 1982;100:1585-1596.

6. Buus DR, Tse DT, Farris BK. Ophthalmic complications of sinus surgery. Ophthalmology. 1990;97:612-619.

7. Montgomery W, Singer M, Hamaker R. Surgical Treatment of Sinus Infections. Diseases of the Nose, Throat, Ear, Head and Neck. Philadelphia: Lea \& Febiger; 1985:224-242.

8. Griffiths JD, Smith B. Optic atrophy following Caldwell-Luc procedure. Arch Ophthalmol. 1975;86:15-18.

9. Hayreh SS, Piegors DJ, Heistad DD. Serotonin-induced constriction of ocular arteries in atherosclerotic monkeys. Implications for ischemic disorders of the retina and optic nerve head. Arch Ophthalmol. 1997;115:220-228.

10. El-Sayed Y, Al-Muhaimeid H. Acute visual loss in association with sinusitis. J Laryngol Otol. 1993;107:840-842.

11. Slavin ML, Glaser J. Acute severe irreversible visual loss with sphenoethmoiditis 'posterior' orbital cellulitis. Arch Ophthalmol. 1987;105:345-348.

12. Hayreh SS, Kolder HE, Weingeist TA. Central retinal artery occlusion and retinal tolerance time. Ophthalmology. 1980;87:75-78. 